\title{
LINER galaxy properties and the local environment
}

\author{
Georgina V. Coldwell, ${ }^{1 \star}$ Sol Alonso, ${ }^{1 \star}$ Fernanda Duplancic $^{1 \star}$ and Valeria Mesa ${ }^{2}$ \\ ${ }^{1}$ Departamento de Geofísica y Astronomía, CONICET, Facultad de Ciencias Exactas, Físicas y Naturales, Universidad Nacional de San Juan, Av. Ignacio de \\ la Roza 590 (O), J5402DCS, Rivadavia, San Juan, Argentina \\ ${ }^{2}$ Instituto Argentino de Nivología, Glaciología y Ciencias Ambientales (IANIGLA-CCT Mendoza, CONICET), Parque Gral San Martín, CC 330, CP 5500, \\ Mendoza, Argentina
}

Accepted 2018 February 12. Received 2018 January 24; in original form 2017 November 10

\begin{abstract}
We analyse the properties of a sample of 5560 low-ionization nuclear emission-line region (LINER) galaxies selected from SDSS-DR12 at low red shift, for a complete range of local density environments. The host LINER galaxies were studied and compared with a well-defined control sample of 5553 non-LINER galaxies matched in red shift, luminosity, morphology and local density. By studying the distributions of galaxy colours and the stellar age population, we find that LINERs are redder and older than the control sample over a wide range of densities. In addition, LINERs are older than the control sample, at a given galaxy colour, indicating that some external process could have accelerated the evolution of the stellar population. The analysis of the host properties shows that the control sample exhibits a strong relation between colours, ages and the local density, while more than 90 per cent of the LINERs are redder and older than the mean values, independently of the neighbourhood density. Furthermore, a detailed study in three local density ranges shows that, while control sample galaxies are redder and older as a function of stellar mass and density, LINER galaxies mismatch the known morphology-density relation of galaxies without low-ionization features. The results support the contribution of hot and old stars to the low-ionization emission although the contribution of nuclear activity is not discarded.
\end{abstract}

Key words: galaxies: active-galaxies: general-galaxies: statistics.

\section{INTRODUCTION}

Galaxies with low-ionization nuclear emission-line regions (LINERs) were first described by Heckman (1980) as a class of extragalactic objects with optical spectra dominated by enhanced lowionization $\mathrm{O} I(\lambda 6300)$ and $\mathrm{N} I I(\lambda 6548,6583)$ lines. Therefore, LINERs were defined by intensity ratios of optical emission lines: (1) $\mathrm{I}\left(\left[\mathrm{O}_{\text {II }}\right] \lambda 3727\right) / \mathrm{I}\left(\left[\mathrm{O}_{\mathrm{III}}\right] \lambda 5007\right) \geq 1$ where $\left[\mathrm{O}_{\mathrm{II}}\right] \lambda 3727$ is used to designate the $\left[\mathrm{O}_{\mathrm{II}}\right] \lambda \lambda 3726,3729$ doublet and (2) $\mathrm{I}\left(\left[\mathrm{O}_{\mathrm{I}}\right] \lambda 6300\right) / \mathrm{I}([\mathrm{O} \mathrm{III}]$ $\lambda 5007) \geq 1 / 3$. These low-ionization features have been found in about 30 per cent of the nearby galaxies (Heckman 1980; Ho, Filippenko \& Sargent 1997), reaching 50 per cent in elliptical passive galaxies (Phillips et al. 1986; Goudfrooij et al. 1994; Yan et al. 2006; Capetti \& Baldi 2011).

Different mechanisms have been proposed to explain the nature of LINER galaxies. Their low-ionization emission lines could be powered by (1) shock-heated gas (Heckman 1980; Dopita \& Sutherland 1995), which is the less likely scenario since the gas velocity dispersion commonly falls below the value required to

^E-mail: georginacoldwell@gmail.com (GVC); solalonsog@gmail.com (SA); fernandaduplancic@gmail.com (FD) explain the observed level of spectral ionization (Ho, Filippenko \& Sargent 2003); (2) photoionization by an active central black hole (Groves, Dopita \& Sutherland 2004), such as an active galactic nucleus (AGN); and (3) stellar photoionization by hot O stars (Filipenko \& Terlevich 1992) or by old post-asymptotic giant branch (post-AGB) stars (Binette et al. 1994; Stasínska et al. 2008).

Several works agree with the assertion that AGN photoionization is the dominant mechanism for LINER emission. In this scenario, Kewley et al. (2006) established that LINERs seem to populate the low-luminosity end of the AGN distribution, where radiatively inefficient accretion flows and external obscuring matter (González-Martín et al. 2009b; Dudik, Satyapal \& Marcu 2009) may cause optical extinction. Thus, LINERs are less luminous than Seyfert galaxies and share similar spectral characteristics, with the remarkable exception that LINERs show enhanced low-ionization $\mathrm{O} I(\lambda 6300)$ and $\mathrm{N}_{\mathrm{II}}(\lambda 6548,6583)$ lines (Heckman 1980). Accordingly, observations at radio (Nagar, Falcke \& Wilson 2005) and X-ray wavelengths (González-Martín et al. 2009a) provide strong support for AGNs as the origin of LINER emission.

Regarding the stellar photoionization hypothesis, several authors suggest that hot post-AGB stars and white dwarfs could provide enough ionization to explain the LINER emission (Binette et al. 1994; Sodré \& Stasińska 1999; Stasínska et al. 2008). Moreover, 
these stellar ionization sources are in spatially extended regions around the nucleus as the $\mathrm{H} \alpha$ and $\mathrm{H} \beta$ brightness profiles do not decrease with $r^{-2}$, as would be expected for the radial dependency of a central ionization source such as an AGN (Sarzi et al. 2010; Yan \& Blanton 2012).

Recently, the surveys MANGA (Mapping Nearby Galaxies at Apache Point Observatory, Bundy et al. 2015) and CALIFA (The Calar Alto Legacy Integral Field Area, Sánchez et al. 2012) have provided valuable spatially resolved observations shedding light on the issues related to the LINER galaxies. Using MANGA, Belfiore et al. (2016) studied the spatially resolved excitation properties of the ionized gas in a sample of 646 galaxies, demonstrating the presence of extended low-ionization emission-line regions on kiloparsec scales. They introduced the acronym LIER, instead of LINER, to highlight that low-ionization lines in most of the galaxies are not nuclear, since post-AGB stars are the most likely candidates for this emission.

Furthermore, Singh et al. (2013) analysed the radial emissionline surface brightness profiles of a sample of LINERs, selected from the CALIFA survey, in comparison to the expected profile for AGNs. Their findings were consistent with emission from extended power sources, in agreement with the work of Papaderos et al. (2013), showing strong evidence in favour of the post-AGB stars hypothesis. Based on the results of the cited works, it seems it would be very useful to distinguish properly between nuclear and extended LINERs, although this does not seem to be trivial. Yan \& Blanton (2012) consider the scale over which the spectrum is taken, and define nuclear LINERs at scales lower than $200 \mathrm{~h}^{-1} \mathrm{pc}$ and extended sources for scales greater than $1 \mathrm{~h}^{-1} \mathrm{kpc}$. In fact, they argued that this distinction strongly depends on the physical aperture of the observation instrument. For SDSS galaxies, with $z \sim 0.1$ most LINERs are spatially extended. Also, the authors state that the host galaxies of nuclear line-emitting regions and those of extended line-emitting regions are largely the same population.

The possibility that several different mechanisms simultaneously power the low-ionization emission lines is also considered. For example, Hubble Space Telescope observations have found that both unresolved nuclear and extended $\mathrm{H} \alpha$ emission are present in the majority of nearby LINERs (Masegosa et al. 2011). Moreover, Graves et al. (2007) found that red-sequence galaxies with LINER emission are younger than their quiescent counterparts, suggesting a connection between the star formation history and the mechanism generating the low-ionization emission. Nevertheless, there is no certainty of the predominant mechanism in LINER galaxies. In addition, Eracleous, Hwang \& Flohic (2010) observed a sample of 35 LINERs, on small scales of order $\sim 200 \mathrm{~h}^{-1} \mathrm{pc}$ from the nucleus, and found that photoionization by post-AGB stars is an important power source, since for more than half of the LINERs it provides more ionizing photons than the AGN and enough ionizing photons to power the emission lines in $1 / 3$ of them. Besides, photoionization from the AGN powers the gas emission on scales of a few tens of parsec, although it is not efficient enough on larger scales, suggesting that different processes could dominate on different scales.

To unravel the nature of the LINER emission, studying environmental factors could provide important clues, since numerous galaxy properties correlate with the density environment. Several studies (Popesso \& Biviano 2006; Coldwell et al. 2009, 2014; Padilla, Lambas \& González 2010) have found that AGN environments do not seem to follow the morphology-density relation proposed by Dressler (1980). If the photoionization from AGNs is one of the mechanisms for the LINER emission, we would expect that, at least, LINERs would follow a similar trend as Seyfert 2 galaxies. Coldwell et al. (2017) analysed the occurrence of SDSSDR7 LINER galaxies with respect to their proximity to galaxy groups. Their main results show that a higher percentage of LINERs populate low-density environments. Besides, if LINERs belong to high virial mass galaxy groups, they do not follow the expected morphology-density relation.

In this paper, we study the behaviour of LINER galaxies compared with a well-defined control sample selected to match the density environment, red shift, luminosity and morphology. The layout of this paper is as follows. In Section 2, we briefly describe the data selection, the LINER classification scheme and the procedure used to construct the control sample. In Section 3, the analysis of galaxy properties and their morphology-density relation are presented. Finally, in Section 4, we discuss the results and draw our conclusions. Throughout this paper, we have assumed a $\Lambda$-dominated cosmology, with $\Omega_{m}=0.3, \Omega_{\lambda}=0.7$ and $H_{0}=100 \mathrm{~km} \mathrm{~s}^{-1} \mathrm{Mpc}^{-1}$.

\section{DATA AND SAMPLE SELECTION}

The sample of galaxies used in this work was drawn from Data Release 12 of the Sloan Digital Sky Survey ${ }^{1}$ (SDSS-DR12, Alam et al. 2015). This survey covers 14555 square degrees of the sky and includes imaging in five broad bands (ugriz), reduced and calibrated using the final set of SDSS pipelines. SDSSDR12 provides spectroscopy for roughly more than two million galaxies including additional galaxy and quasar spectra from the SDSS-III Baryon Oscillation Spectroscopic Survey (BOSS, Dawson et al. 2013).

All catalogue data were obtained through SQL queries in CASJoBs. ${ }^{2}$ We selected galaxies with spectroscopic information and extinction-corrected model magnitudes, which is more appropriate for extended objects (e.g. galaxies) and also this provides more robust galaxy colours. The magnitudes were $k$-corrected using the empirical $k$-corrections presented by O'Mill et al. (2011).

The physical properties of the galaxies used in this analysis were taken from MPA-JHU. ${ }^{3}$ The procedures to estimate parameters such as stellar masses, emission-line fluxes, stellar age indicators, etc. are based on the methods of Brinchmann et al. (2004), Tremonti et al. (2004) and Kauffmann et al. (2003).

\subsection{LINER sample}

The LINERs were selected using the publicly available emissionline fluxes corrected for optical reddening using the Balmer decrement and the Calzetti et al. (2000) dust curve. We assume $R_{V}=A_{V} / E(B-V)=3.1$ and an intrinsic Balmer decrement $(\mathrm{H} \alpha / \mathrm{H} \beta)_{0}=3.1$ (Osterbrock \& Miller 1989). The line measurements are described by Tremonti et al. (2004) and Brinchmann et al. (2004).

To select LINERs from the emission-line galaxy sample, we included only galaxies with signal-to-noise $>2$ for all the lines in the current analysis. This conservative criterion only moderately reduces the size of the sample and ensures we have a more reliable selection of LINER objects. Following Coldwell et al. (2017), the sample was restricted to the red-shift range $0.04<z<0.1$

\footnotetext{
${ }^{1}$ https://www.sdss3.org/dr12/

${ }^{2}$ http://skyserver.sdss.org/casjobs/

${ }^{3}$ http://www.sdss.org/dr12/spectro/galaxy_mpajhu/
} 


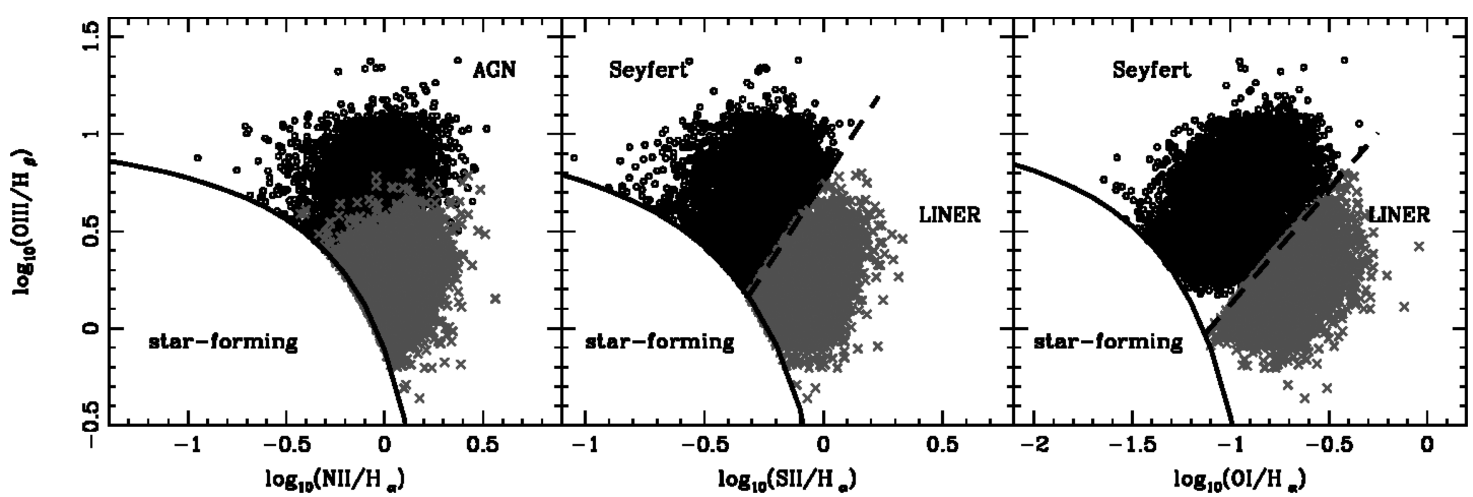

Figure 1. BPT diagrams of the selection criteria defined by Kewley et al. (2006) to classify emission-line galaxies as Seyfert or LINER. The panels show the line ratios $\log \left([\mathrm{O}\right.$ III $] / \mathrm{H} \beta$ ) versus $\log \left(\left[\mathrm{N}_{\text {II }} / \mathrm{H} \alpha\right]\right)$ (left), $\log \left(\left[\mathrm{O}_{\text {III }}\right] / \mathrm{H} \beta\right.$ ) versus $\log \left(\left[\mathrm{S}_{\mathrm{II}} / \mathrm{H} \alpha\right]\right.$ (centre) and $\log \left(\left[\mathrm{O}_{\text {III }}\right] / \mathrm{H} \beta\right)$ versus $\log ([\mathrm{O}$ I/H $\alpha]$ (right). Seyferts are indicated by black dots and LINER galaxies by grey crosses. The solid lines separate star-forming galaxies from AGNs and the dashed lines represent the Seyfert/LINER demarcation.

and we distinguished Seyfert 2, LINER and star-forming galaxies using the three standard line-ratio diagrams (Baldwin, Phillips \& Terlevich 1981, BPT). Thus, we adopted the empirical demarcation lines from Kewley et al. $(2001,2006)$ for the AGN/starburst and for the LINER/Seyfert separations.

Finally, we obtained an effective sample of 5581 LINER objects. It is important to emphasize that the spectral resolution of the circular fibre used to measure SDSS-DR12 spectra, at the mean red shift of this sample, allows us to select mainly extended LINERs, although nuclear LINERs could be hidden in our sample. The separated samples and the selection criteria are shown in the three BPT diagrams of Fig. 1.

\subsection{Control sample}

In a recent work, Coldwell et al. (2017) analysed a sample of SDSSDR7 LINER galaxies with respect to their proximity to galaxy groups taking into account that the physical properties of LINER host galaxies have morphologies, colours and ages corresponding to galaxies residing in the central regions of galaxy groups or clusters. In that study, the authors used a well-defined control sample to obtain strong conclusions for the relation between the low-ionization features of LINER galaxies and the high-density environment. However, the results show that LINERs are more likely to populate lowdensity environments despite their morphology, in comparison with the neighbouring galaxies with identical morphological features but no LINER emission.

From a different perspective, in this paper we explore the properties of LINER galaxies, taken from SDSS-DR12, over a complete range of densities. Furthermore, with the aim of revealing the relation between the low-ionization emission-line mechanisms and the environment, a suitable control sample of galaxies was used for comparison. In this sense, by using SDSS mock galaxy catalogues built from the Millennium Simulation, Perez, Tissera \& Blaizot (2009) showed that a suitable control sample of galaxies in pairs should be selected (at least) with matched distributions of red shift, morphology, stellar mass and local density environment.

Bearing this in mind, we applied a similar criterion to construct our control sample by selecting galaxies without low-ionization emission features from SDSS-DR12 with matched distributions of (i) red shift, (ii) luminosity for the absolute magnitude in the $r$ band, (iii) concentration index $C$ defined as the ratio of the radii containing
90 per cent and 50 per cent of the Petrosian flux, respectively, and (iv) the local density, $\Sigma_{5}$, defined as

$\Sigma_{5}=\frac{5}{\pi d_{5}^{2}}$,

where $d_{5}$ corresponds to the projected distance of the fifth neighbour brighter than $M_{r}<-20.5$. This two-dimensional density estimator uses the red-shift information to reduce the projection effects and is useful for characterizing the local galaxy density. The advantage of this method is that it uses a systematically larger scale in lowerdensity regions, which improves sensitivity and precision at low densities. We chose a fixed velocity interval of $\Delta V=1000 \mathrm{~km} \mathrm{~s}^{-1}$ to compute the local density, which corresponds to galaxies within $\sim 3 \sigma$ from the centre of a galaxy cluster (Balogh et al. 2004) and this allows the inclusion of galaxies in a system with a large velocity dispersion.

In addition, the concentration index $C$ can be correlated with the morphological characteristic of galaxies (Shimasaku et al. 2001; Strateva et al. 2001). Thus, galaxies with a de Vaucouleurs profile have $C \sim 3.3$ and disc galaxies have a concentration index $C \sim 2.4$. Fig. 2 shows normalized distributions of red shift, luminosity, morphology and local density for both LINERs and the control sample. The carefully selected control sample comprises 5553 galaxies. By matching these parameters, we are able to detect, for the remaining free parameters, the distinctive features of LINERs likely associated with the low-ionization emission mechanisms.

\section{ANALYSIS}

Different studies have demonstrated that the host galaxies of LINERs have a bulge-type morphology that is more massive and luminous than that of a general galaxy sample (Yan et al. 2006; Kewley et al. 2006; Capetti \& Baldi 2011; Coldwell et al. 2017). These early-type galaxies are commonly found in dense galaxy environments, as suggested by the morphology-density relation (Dressler 1980; Domínguez, Muriel \& Lambas 2001). However, Coldwell et al. (2017) have shown that the probability of finding LINER galaxies in rich galaxy groups is lower than that for a low-density environment. Thus, LINERs do not seem to follow the expected morphology-density relation.

From a wide perspective, in this work we use a complete range of densities to explore the dependence of the host galaxy properties with the low-ionization features and the environment, since only 

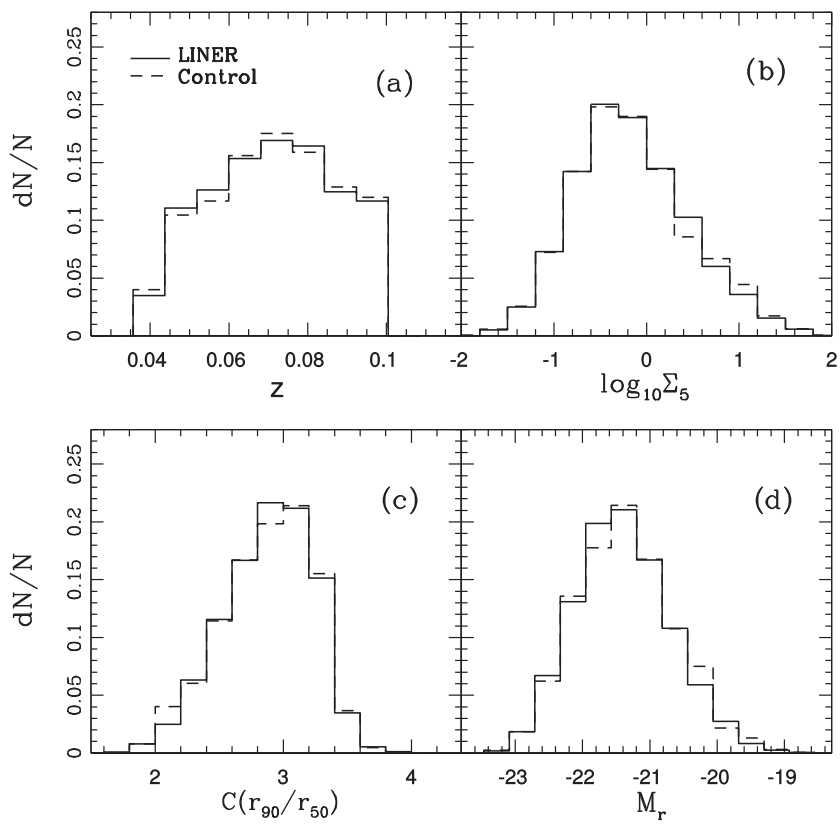

Figure 2. Normalized distributions of galaxy properties and the local density for LINERs (solid lines) and the control sample (dashed lines).

LINERs close to galaxy groups were analysed by Coldwell et al. (2017). Moreover, exhaustive statistical studies of the LINERs environmental dependence have not been done until now.

\subsection{Colours and stellar age populations of host galaxies}

Galaxy colours can be used as estimators of their evolution. So, in clusters, a large fraction of red galaxies indicates an old population of galaxies with a low star-formation rate. These evolved sources, as a consequence of dynamical processes such as interactions, rampressure stripping and strangulation (Gunn \& Gott 1972; Larson, Tinsley \& Caldwell 1980; Balogh, Navarro \& Morris 2000), are typically elliptical galaxies. In contrast, galaxies in poor groups or in the field are bluer and with a stronger star formation rate, indicating young sources.

In addition, the break index $D_{\mathrm{n}}(4000)$ (Kauffmann \& Haehnelt 2002), defined as the ratio of the average flux density in the narrow continuum bands (3850-3950 and 4000-4100 $\AA$ ), is suitably correlated to the mean age of the stellar population in a galaxy. It can be used to estimate the star formation rate (Brinchmann et al. 2004), since the majority of star formation takes place preferentially in galaxies with a low $D_{\mathrm{n}}(4000)$.

In this section, we analyse in detail the colours and age indicators of the galaxies in the samples. The left-hand panels of Fig. 3 show the distributions of stellar age populations and colours, given by $D_{\mathrm{n}}(4000)$ and $M_{g}-M_{r}$ respectively, of the LINER and control galaxy samples. Also we plot the distributions of these parameters for galaxies in the SDSS Main Galaxy Sample (MGS) in the redshift range $0.04<z<0.1$. Both samples, LINERs and controls, are redder and older than MGS galaxies. However, a significant difference between them is detected. An evident lack of young and blue galaxies with LINER emission can be observed in both distributions in comparison with the control sample.

To quantify this effect, we calculated the fractions of galaxies with stellar age populations $D_{\mathrm{n}}(4000)>1.53$ and colours $M_{g}-M_{r}>0.76$. These constraints correspond to the mean values of these parameters for the MGS in the red-shift range considered in this work. Hence, we estimate that 94 per cent of LINER galaxies have older stellar populations and redder colours than the average, while this percentage decreases up to 78 percent for the control sample galaxies.

In addition, the right-hand panels of Fig. 3 show that LINERs are older and have redder colours than the corresponding control sample, independently of their local density. In contrast, galaxies in the control sample have a larger spread of colours and the ages of their stellar population, which are bluer and younger at lower densities.

Furthermore, the colours and stellar age show clearly that LINERs belong to a more evolved galaxy population for several ranges of local galaxy density, although Fig. 3 suggests there is a difference between these two parameters for both samples. To analyse this effect, we quantified any excess of the old stellar age population with respect to their galaxy colours by calculating the fraction of galaxies with $D_{\mathrm{n}}(4000)>1.53$. In Fig. 4 , it is possible to appreciate the dependence of colours with the age of galaxies, as is expected from galaxy evolution. Thus, evolved galaxies are redder than the youngest ones. Nevertheless, the fraction of old galaxies is higher for bluer LINERs, suggesting that these sources could have experienced some ageing of their stellar population. For galaxies redder than $M_{g}-M_{r} \sim 0.9$, the fraction of galaxies older than the mean is $\approx 100$ per cent and this is indistinguishable between the LINER and control samples. The error bars in the figures were calculated by using bootstrap error resampling (Barrow, Bhavsar \& Sonoda 1984).

The finding shows that LINER host galaxies belong to the most evolved galaxy population observed, independently of the local density environment. The recent works of Singh et al. (2013) and Belfiore et al. (2016) suggest that the ubiquitous presence of this hot and evolved stellar population in extended LINERs strongly supports the assumption of post-AGB stars as the mechanism responsible for powering the low-ionization emission lines.

\subsection{Morphology-density relation}

The relationship between density and morphology has been demonstrated by several authors. Evolved elliptical galaxies inhabit highdensity environments while blue disc galaxies commonly reside in low-density regions (Dressler 1980; Domínguez et al. 2001). However, for AGNs, different studies (Popesso \& Biviano 2006; Coldwell et al. 2009, 2014; Padilla et al. 2010) have found that these objects do not follow the expected morphology-density relation of galaxies without nuclear activity. By exploring this relation for LINER host galaxies, it could be possible to obtain clues about the existence of nuclear activity in the centre of extended LINERs.

In this section, we estimate the fraction of LINER and control galaxies older and redder than the average values $\left(D_{\mathrm{n}}(4000)>1.53\right.$ and colours $\left.M_{g}-M_{r}>0.76\right)$ as a function of the local density, $\Sigma_{5}$. In Fig. 5, we show these fractions for both samples. Notice that approximately 100 per cent of LINER galaxies are redder and older than the mean values, in agreement with the results of Fig. 3. In addition, this result is not dependent on the local density, within the error bars. In contrast, the control sample shows a decreasing fraction of evolved galaxies at lower densities, as expected for the well-known morphology-density relation.

It is important to notice that the mentioned differences between both samples could be directly related to the ionization mechanisms, since the control sample was selected to match the luminosity, 

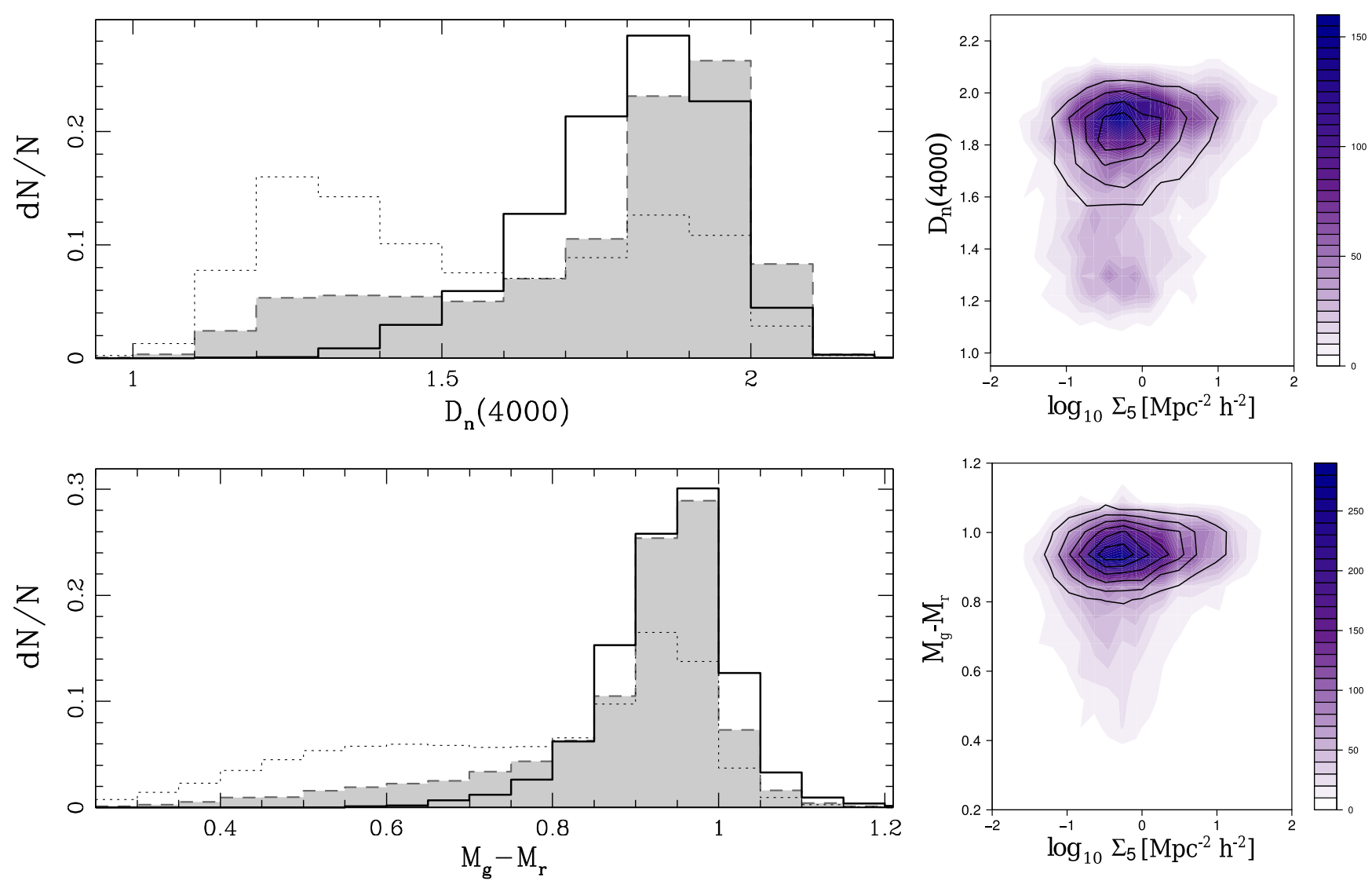

Figure 3. Left: Normalized distributions of stellar age population parameter $D_{\mathrm{n}}(4000)$ (top) and colours $M_{g}-M_{r}$ (bottom) for LINERs (solid lines) and the control sample (shaded histograms). The dotted lines correspond to the MGS in the red-shift range of the targets. Right: $D_{\mathrm{n}}(4000)$ versus local density $\Sigma_{5}$ (top) and $M_{g}-M_{r}$ versus $\Sigma_{5}$ (bottom) for LINERs (solid contours) and the control sample (coloured density maps).

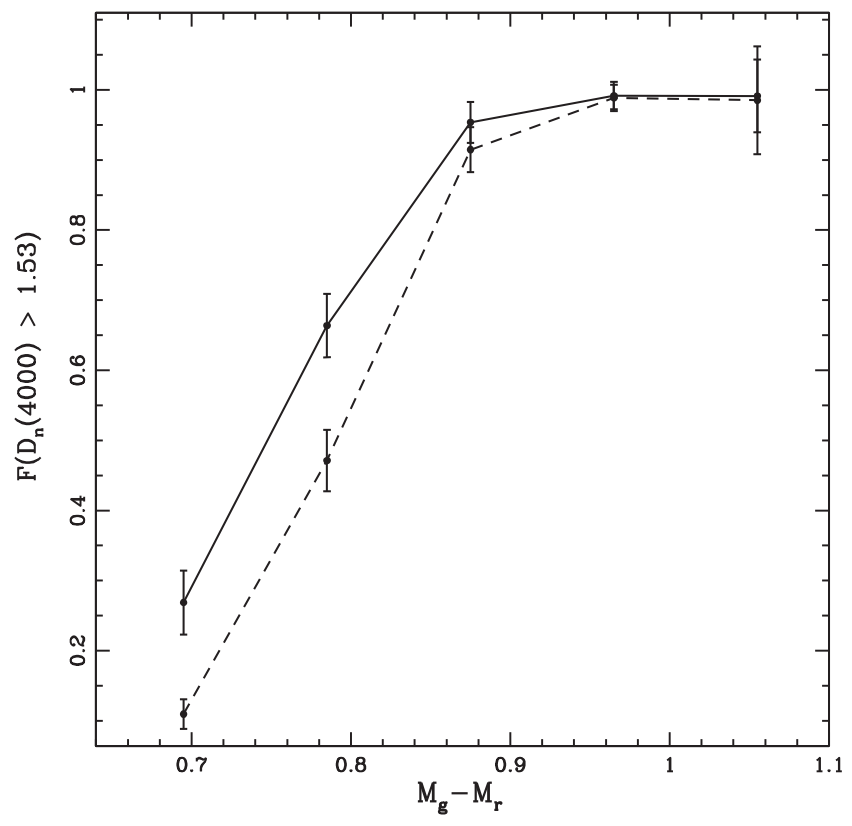

Figure 4. Fraction of old $\left(D_{\mathrm{n}}(4000)>1.53\right)$ galaxies as a function of the colours $M_{g}-M_{r}$. The solid line corresponds to the LINER sample and the dashed line to the control sample.

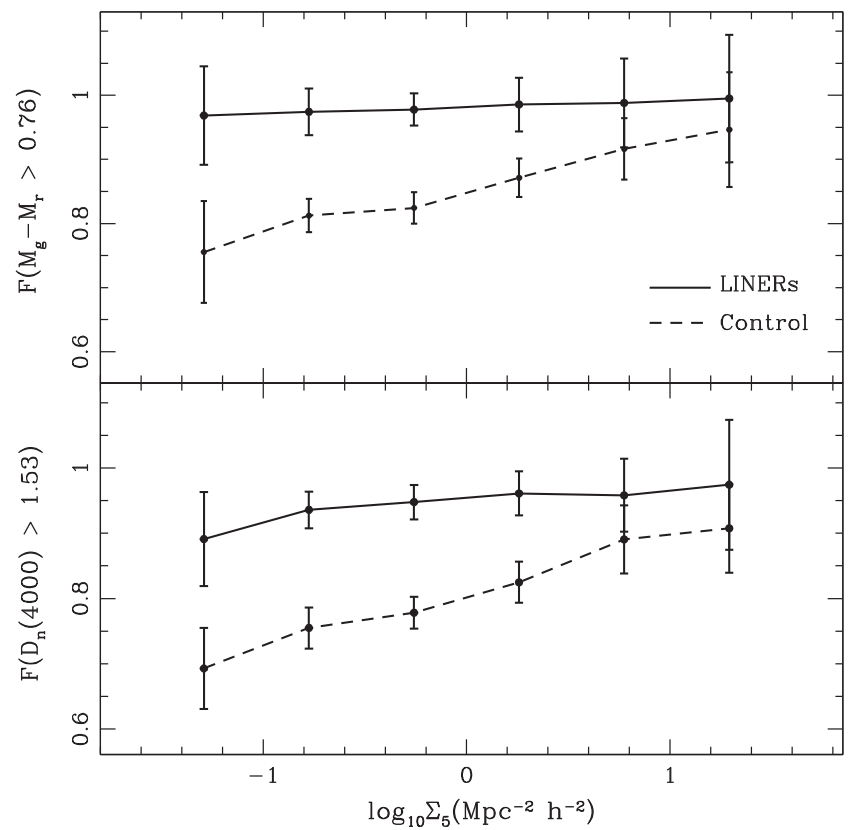

Figure 5. Fraction of red $\left(M_{g}-M_{r}>0.76\right)$ and old $\left(D_{\mathrm{n}}(4000)>1.53\right)$ galaxies as a function of the local density $\Sigma_{5}$. The solid lines correspond to the LINER sample and the dashed lines to the control sample. 


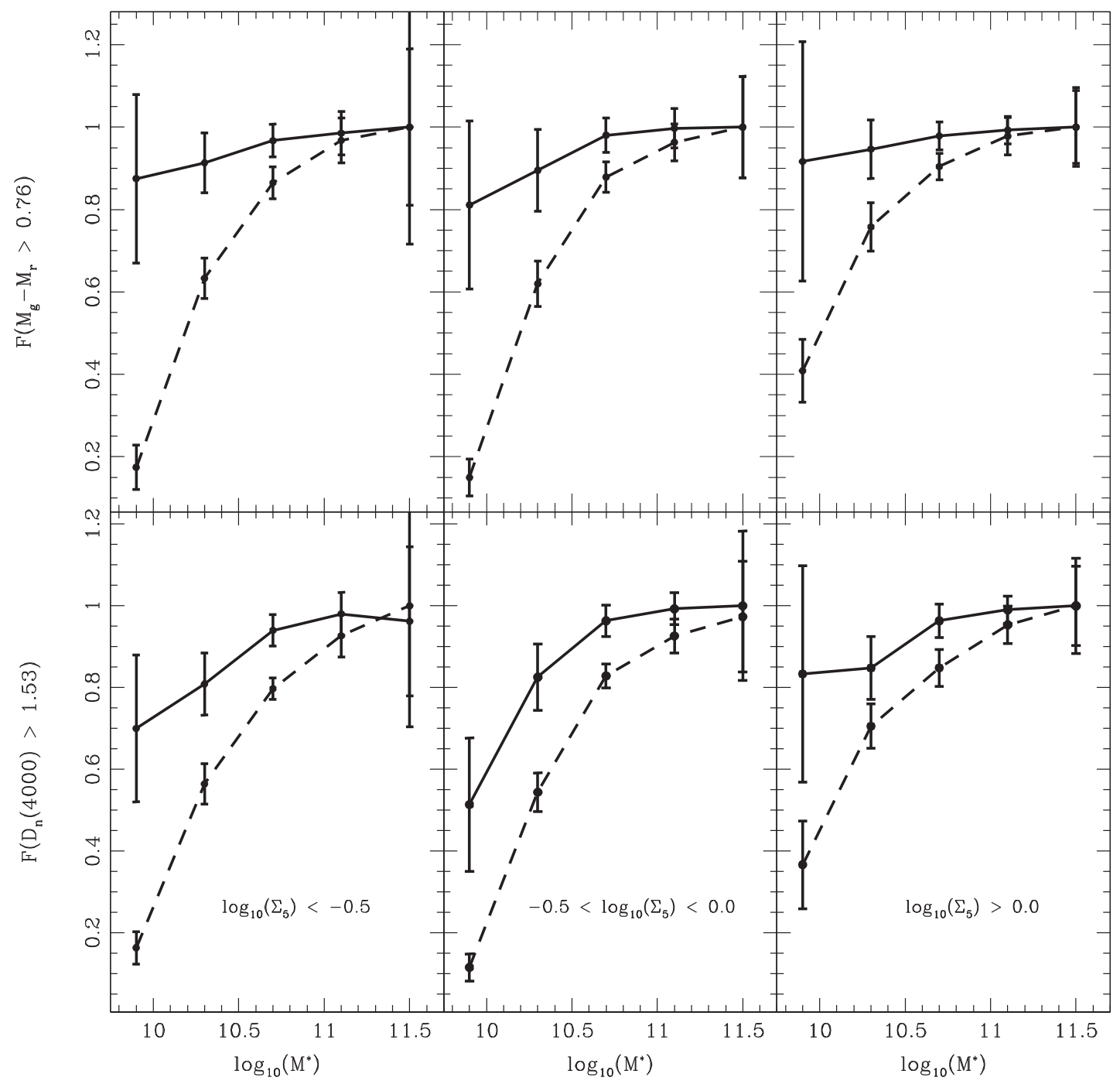

Figure 6. Fraction of red $\left(M_{g}-M_{r}>0.76\right)$ and old $\left(D_{\mathrm{n}}(4000)>1.53\right)$ galaxies as a function of the stellar mass $M^{*}$, in logarithmic scale, for three different local density ranges. The solid lines correspond to the LINER sample and the dashed lines to the control sample.

concentration index, red shift and local density of the LINERs. Hence, the colours and stellar age populations are more sensitive to the physical processes involved, as indicated by their different stages of galaxy evolution.

On the other hand, the stellar mass parameter correlates with colours, density and ages of galaxies. Thus, we expect lower values of stellar mass for blue and young typical galaxies (without special features like an AGN). We examined the trends for the fraction of red and old galaxies with respect to the stellar mass for both samples in three different density ranges. We used the parameter $M^{*}$, in a logarithmic scale, as previously determined by Kauffmann et al. (2003). The method relies on spectral indicators relating to the stellar age population and the fraction of stars formed in recent bursts.

Fig. 6 shows the fraction of red $\left(M_{g}-M_{r}>0.76\right)$ and old $\left(D_{\mathrm{n}}(4000)>1.53\right)$ galaxies as a function of the stellar mass $M^{*}$ for three bins for local density given by $\log _{10} \Sigma_{5}<-0.5$, $-0.5<\log _{10} \Sigma_{5}<0.0$ and $\log _{10} \Sigma_{5}>0.0$. These three ranges were selected to include approximately 33 percent of the sample each. They represent the low-, intermediate- and high-density en- vironments, respectively. From this figure, it is possible to observe a clear trend for galaxies in the control sample to become redder and older with increasing stellar mass. Moreover, these fractions are larger in the highest density range. However, LINERs seem to be less sensitive to the host stellar mass. Their stellar age population shows a weak tendency to be younger for lower values of stellar mass, at low and intermediate densities, although the fraction of the $D_{\mathrm{n}}(4000)$ parameter is almost independent of the stellar mass at high densities.

The noticeable effect for galaxy colour trends observed in the upper panels of Fig. 6, where the fractions remain nearly constant for the whole range of $M *$ (within the error bars) independently of the local density environment, gives strong support to the lack of dependence between galaxy morphology and spatial density for LINER galaxies. These results present a good agreement with the work of Coldwell et al. (2017). Furthermore, the observed trends for LINER galaxies are thoroughly consistent with that found for AGNs by Popesso \& Biviano (2006), Coldwell et al. (2009, 2014) and Padilla et al. (2010). Despite that extended LINERs have an old stellar population, consistent with post-AGB stars, which could be 
the main ionizing mechanism, we cannot discount that there is an AGN in the centre of these galaxies.

\section{DISCUSSION}

We derived a large LINER galaxy catalogue using the SDSSDR12 survey, comprising 5560 objects in the red-shift range $0.04<z<0.1$. Taking into account the observational constraints and the median red shift of the galaxy sample, this is a sample of extended LINERs. To unveil the true environmental density dependence of the main characteristics of galaxies hosting a LINER and obtain clues about the mechanisms involved in the generation of low-ionization emission lines, we constructed a control sample of galaxies, without the observed low-ionization features, matched in red shift, luminosity, morphology and local density. This control sample contains 5553 galaxies.

In this analysis, we use a wide range of densities given by the local density estimator $\Sigma_{5}$ to explore the dependence of this parameter on the stellar age populations and galaxy colours. The distributions of $D_{\mathrm{n}}(4000)$ and $M_{g}-M_{r}$ show that both LINER and control samples are older and redder than galaxies in the SDSS MGS in the red-shift range considered in this work. Nevertheless, a significant difference is seen between the colours and stellar age population of the LINERs and the control sample. LINERs are noticeably older and redder than the control sample and this remains observable over the entire density range. In addition, an excess of old galaxies is detected for LINERs with respect to the control sample, at a given colour bluer than $M_{g}-M_{r} \approx 0.9$. This finding could suggest that LINERs have experienced some process that accelerates the evolution of their stellar population.

To study the dependence of the LINER host properties on the environmental density, we calculated the fraction of red $\left(M_{g}-M_{r}>0.76\right)$ and old $\left(D_{\mathrm{n}}(4000)>1.53\right)$ galaxies with respect to the local density. We found a remarkable difference between the behaviour of LINERs and the control sample. While the control galaxies show an increasing fraction of red and old galaxies with increasing local density, approximately more than 90 per cent of the LINERs are redder and older than these mean values, independently of the neighbourhood density. This result implies that LINERs do not follow the known morphology-density relation for galaxies without low-ionization features.

In a more detailed analysis of the galaxy properties, we estimated the fraction of red colours and old stellar population as a function of the stellar mass of galaxies $\left(\log _{10}\left(M^{*}\right)\right)$ in three different ranges of local density. This variable strongly correlates with the processes involved in galaxy evolution and density. The observed trends of the control sample clearly represent the expected morphology-density relation. Thus, the fractions of both colours and stellar age population increase at larger values of stellar mass. In addition, these fractions are larger for the higher density ranges. In contrast, the fractions of $D_{\mathrm{n}}(4000)>1.53$ for LINER galaxies are higher than that corresponding to the control sample. Moreover, while a weak trend of increasing fractions can be observed at low- and intermediate-density ranges, this fraction does not depend on the host stellar mass in the highest density range. Surprisingly, the fraction of $M_{g}-M_{r}$ LINER galaxy colours, redder than the mean value, is independent of the stellar mass and it remains constant, close to 100 percent, for the three local density ranges.

Summarizing the main results, we highlight that LINER galaxies show a different behaviour than the control galaxy sample by analysing their colours and stellar age population. Certainly,
LINERs have redder colours and older stellar populations independently of their stellar mass and the local density. This finding is in agreement with the works of Eracleous et al. (2010), Yan \& Blanton (2012), Singh et al. (2013) and Belfiore et al. (2016), and it suggests there is a significant contribution from old and red, hot post-AGB stars as a driver of the photoionization. However, although the postAGB hypothesis appears the most viable mechanism for extended LINERs, these objects also exhibit features consistent with the presence of a central AGN, like the morphology-density relation. The observational constraints make it difficult to draw definite conclusions. However, it is feasible that different mechanisms coexist at different scales.

\section{ACKNOWLEDGEMENTS}

We would like to thank the anonymous referee for the suggestions that helped to improve this paper. GVC acknowledges technical support from Dr Rodrigo Romero and Estudio Conturso \& Asoc. This work was supported in part by the Consejo Nacional de Investigaciones Científicas y Técnicas de la República Argentina (CONICET), the Consejo Nacional de Investigaciones Científicas, Técnicas y de Creación Artística de la Universidad Nacional de San Juan (CICITCA) and the Secretara de Estado de Ciencia, Tecnología e Innovación del Gobierno de San Juan (SECITI).

Funding for SDSS-III has been provided by the Alfred P. Sloan Foundation, the participating institutions, the National Science Foundation, and the US Department of Energy Office of Science. The SDSS-III web site is http://www.sdss3.org/. SDSS-III is managed by the Astrophysical Research Consortium for the participating institutions of the SDSS-III Collaboration, including the University of Arizona, the Brazilian Participation Group, Brookhaven National Laboratory, Carnegie Mellon University, University of Florida, the French Participation Group, the German Participation Group, Harvard University, the Instituto de Astrofisica de Canarias, the Michigan State/Notre Dame/JINA Participation Group, Johns Hopkins University, Lawrence Berkeley National Laboratory, Max Planck Institute for Astrophysics, Max Planck Institute for Extraterrestrial Physics, New Mexico State University, New York University, Ohio State University, Pennsylvania State University, University of Portsmouth, Princeton University, the Spanish Participation Group, University of Tokyo, University of Utah, Vanderbilt University, University of Virginia, University of Washington and Yale University.

\section{REFERENCES}

Alam S. et al., 2015, ApJS, 219, 12

Baldwin J. A., Phillips M. M., Terlevich R., 1981, PASP, 93, 5

Balogh M. L., Navarro J. F., Morris S. L., 2000, ApJ, 540, 113

Balogh M. et al., 2004, MNRAS, 348, 1355

Barrow J. D., Bhavsar S. P., Sonoda B. H., 1984, MNRAS, 210, 19

Belfiore F. et al., 2016, MNRAS, 461, 3111

Binette L., Magris C. G., Stasinska G., Bruzual A. G., 1994, A\&A, 292, 13

Brinchmann J., Charlot S., White S. D. M., Tremonti C., Kauffmann G., Heckman T., Brinkmann J., 2004, MNRAS, 351, 1151

Bundy K. et al., 2015, ApJ, 798, 7

Calzetti D., Armus L., Bohlin R. C., Kinney A. L., Koornneef J., StorchiBergmann T., 2000, ApJ, 533, 682

Capetti A., Baldi R. D., 2011, A\&A, 529, 126

Coldwell G. V., Lambas D. G., Söchting I. K., Gurovich S., 2009, MNRAS, 399, 88

Coldwell G. V., Gurovich S., Diaz Tello J., Söchting I. K., Lambas D. G., 2014, MNRAS, 437, 1199 
Coldwell G. V., Pereyra L., Alonso S., Donoso E., Duplancic F., 2017, MNRAS, 467, 3338

Dawson K. S. et al., 2013, AJ, 145, 10

Domínguez M., Muriel H., Lambas D. G., 2001, AJ, 121, 1266

Dopita M. A., Sutherland R. S., 1995, ApJ, 455, 468

Dressler A., 1980, ApJ, 236, 351

Dudik R. P., Satyapal S., Marcu D., 2009, ApJ, 691, 1501

Eracleous M., Hwang J. A., Flohic H. M. L. G., 2010, ApJ, 711, 796

Filippenko A. V., Terlevich R., 1992, ApJ, 397, L79

González-Martín O., Masegosa J., Márquez I., Guainazzi M., JiménezBailón E., 2009a, A\&A, 506, 1407

González-Martín O., Masegosa J., Márquez I., Guainazzi M., 2009b, ApJ, 704,1570

Goudfrooij P., Hansen L., Jorgensen H., Norgaard-Nielsen H., 1994, A\&AS, 105,341

Graves G. J., Faber S. M., Schiavon R. P., Yan R., 2007, ApJS, 671, 243

Groves B. A., Dopita M. A., Sutherland R. S., 2004, ApJS, 153, 75

Gunn J. E., Gott J. R., III, 1972, ApJ, 176, 1

Heckman T. M., 1980, A\&A, 87, 152

Ho L. C., Filippenko A. V., Sargent W. L. W., 1997, ApJS, 112, 391

Ho L. C., Filippenko A. V., Sargent W. L. W., 2003, ApJ, 583, 159

Kauffmann G., Haehnelt M. G., 2002, MNRAS, 332, 529

Kauffmann G. et al., 2003, MNRAS, 341, 33

Kewley L. J., Dopita M. A., Sutherland R. S., Heisler C. A., Trevena J., 2001, ApJ, 556, 121

Kewley L. J., Groves B., Kauffmann G., Heckman T. M., 2006, MNRAS, 372,961

Larson R. B., Tinsley B. M., Caldwell C. N., 1980, ApJ, 237, 692
Masegosa J., Márquez I., Ramirez A., González-Martn O., 2011, A\&A, 527, 23

Nagar N. M., Falcke H., Wilson A. S., 2005, A\&A, 435, 521

O’Mill A. L., Duplancic F., García Lambas D., Sodré L., Jr, 2011, MNRAS, 413, 1395

Osterbrock D. E., Miller J. S., 1989, Proc. IAU Symp. 134. Active Galactic Nuclei. Kluwer Academic Publishers, Dordrecht

Padilla N., Lambas D. G., González R., 2010, MNRAS, 409, 936

Papaderos P. et al., 2013, A\&A, 555, 1

Perez J., Tissera P., Blaizot J., 2009, MNRAS, 397, 748

Phillips M. M., Jenkins C. R., Dopita M. A., Sadler E. M., Binette L., 1986, AJ, 91, 1062

Popesso P., Biviano A., 2006, A\&A, 460, L23

Sánchez S. F. et al., 2012, A\&A, 538, 8

Sarzi M. et al., 2010, MNRAS, 402, 2187

Shimasaku K. et al., 2001, AJ, 122, 1238

Singh R. et al., 2013, A\&A, 558, A43

Sodré L., Stasińska G., 1999, A\&A, 345, 391

Stasínska G., Vale Asari N., Cid Fernandes R., Gomes J. M., Schlickmann M., Mateus A., Schoenell W., Sodré L., Jr, 2008, MNRAS, 391, 29

Strateva I. et al., 2001, AJ, 122, 1861

Tremonti C. et al., 2004, ApJ, 613, 898

Yan R., Blanton M. R., 2012, ApJ, 747, 61

Yan R., Newman J. A., Faber S. M., Konidaris N., Koo D., Davis M., 2006, ApJ, 648, 821

This paper has been typeset from a $\mathrm{TE}_{\mathrm{E}} \mathrm{X} / \mathrm{AT} \mathrm{T} \mathrm{X}$ file prepared by the author. 